\title{
On some features of geoacoustic emission signals before earthquakes
}

\author{
Albert Shcherbina ${ }^{1}$ \\ ${ }^{1}$ Institute of Cosmophysical Research and Radio Wave Propagation FEB RAS, Paratunka, Russia
}

\begin{abstract}
Geoacoustic emission signal amplitudes and arrival directions were analyzed during background periods and earthquake preparation. It was determined that during seismic event activation, geoacoustic emission directivity anisotropy increases with the increase of signal amplitude. Taking this feature into account, geoacoustic emission properties were estimated by the superposition method before 2653 earthquakes which occurred within the period from 01.01.2008 to 01.09.2016. It was determined that depending on the type of the signals under consideration, it is possible to detect geoacoustic emission anomalous behavior at the distances of more than $25 \%$ and $65 \%$ of the precursor manifestation zone radius suggested by I.P. Dobrovol'skiy.
\end{abstract}

\section{Introduction}

The long-term investigations carried out in Kamchatka show that significant increase of geoacoustic emission intensity and its directivity change are observed before strong seismic events. This acoustic effect manifests the most brightly in the kilohertz frequency range and is determined by rock deformations at observation sites at the distances of hundreds of kilometers from the epicenters of preparing earthquakes [1,2].

Geoacoustic emission is registered in the frequency range up to $11 \mathrm{kHz}$ by a highfrequency combined receiver allowing us to record acoustic pressure and three mutually orthogonal components of its gradient at one space point. The receiver is installed by the bottom of Mikizha Lake that is located in a seismically active zone of Kamchatka peninsula, near Paratunka village. The developed methods for signal processing allow us to distinguish geoacoustic pulses in automatic regime, to determine their amplitude, repetition frequency and the direction to a source [3]. Since 2008 all the collected data are regularly added to a data base.

One of the problems that is interesting to solve is the comparison of the data series on earthquakes taking into account their energy parameters and distances with the data series on geoacoustic field responses in the measurement region. The superposition method gives good results in this problem solution [4].

\section{Data analysis and results}


To apply the superposition method effectively, we need to form the data series characterizing the geoacoustic field state the most accurately. In the paper mentioned above, the acoustic pressure averaged signal was analyzed before 46 earthquakes. In the result, differentiating features of geoacoustic signal anomalous state were detected. Thus, we received a formalized time series (massive) with the sampling period of one minute in which units indicate the disturbance presence at a definite time moment and the zeros indicate its absence.

The suggested method for series formation requires much of manual operation, consequently, it is difficult to apply it for the processing of a large quantity of data (about several thousands of cases). To solve this problem, we will consider the available information on geoacoustic emission signals.

Geoacoustic pulse information, stored in the data bases, allows us to determine the integral $\Omega(t)$ and differential $D(\alpha, t)$ geoacoustic activity. The first value is the pulse repetition frequency depending on time, the second value is the direction distribution of pulse repetition frequency $d \alpha$ in the horizontal plane. Moreover, there is a possibility to select the signals in the required interval of amplitudes $d A$.

Fig. 1 illustrates typical examples of directivity azimuthal diagrams. It is clear from the graphs that during seismic event preparation, significant increase of pulse repetition frequency and, that is more important for this work, the increase of the root-mean-square deviation (RMS) of the directivity diagram are observed. On the presented examples, the diagram RMS corresponding to the background period is of the order $5 \times 10^{-5} \mathrm{imp}$./degr.s., that for the disturbed period is about $2 \times 10^{-4} \mathrm{imp}$./degr.s.
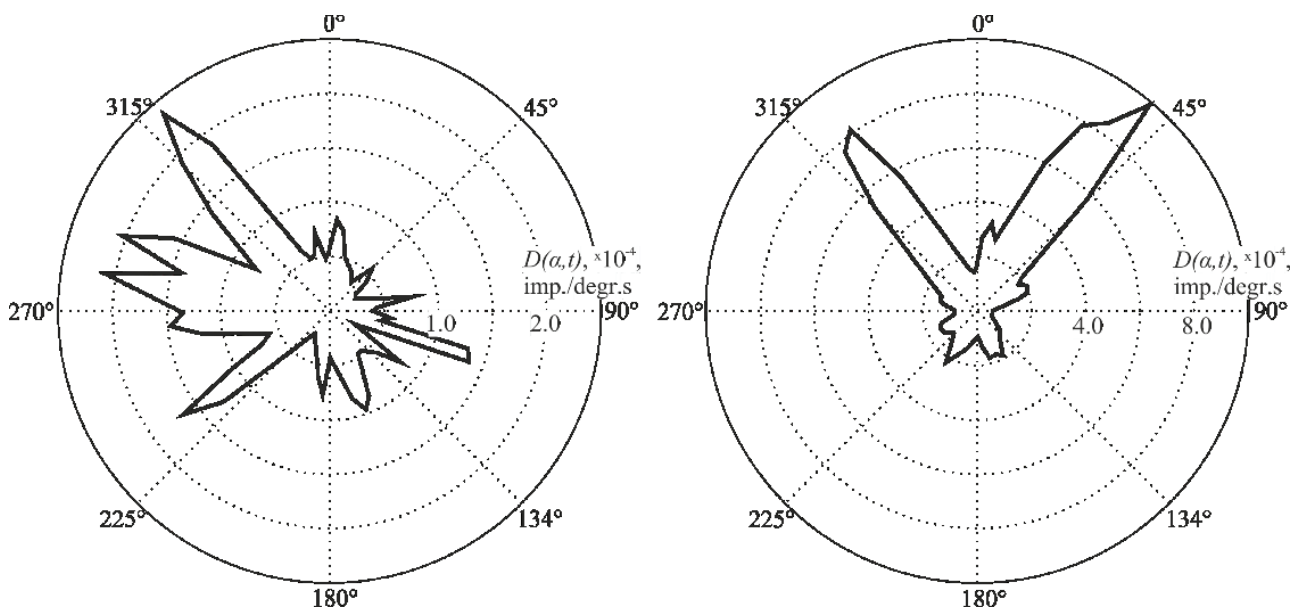

Fig. 1. Examples of directivity azimuthal diagrams $D(\alpha, t)$ : to the left is the background period, to the right is the geoacoustic emission registered before the earthquake occurred on 11.07.2017 at 18:09 UT (energy class is 10.3, distance to the epicenter is $79 \mathrm{~km}$ ).

Analysis of the geoacoustic emission directional properties before 77 seismic events, which occurred within the period from 03.11.2008 to 30.01.2016, showed that geoacoustic signal anisotropy increases as pulse amplitude increases. To demonstrate this, we plot a histogram of geoacoustic pulse amplitude distribution (Fig. 2a). Then we divide the obtained distribution at a conditional point $x$ on the abscissa axis, for example at the value of $10 \%$ from the signal dynamic range (Fig. 2c). The relation of azimuthal distribution diagram RMS for the pulses with the amplitude exceeding this threshold to the signal distribution diagram RMS of less amplitude is determined by the expression

$$
\sigma^{\prime}(x)=\frac{\sigma_{h}(x)}{\sigma_{l}(x)}
$$


where:

$\sigma_{h}(x)=\sigma\left(D(\alpha, t)_{d A \geq x}\right)$ is the azimuthal distribution diagram RMS for the pulses with amplitude exceeding or equal to the threshold $x$,

$\sigma_{l}(x)=\sigma\left(D(\alpha, t)_{d A<x}\right)$ is the same but for the pulses with the amplitude less than the threshold $x$,

$d A$ is the geoacoustic pulse amplitude range applied in the calculation.

Having re-counted this expression for all the points $x$ of the signal amplitude dynamic range, we can plot a curve of the relations of diagram RMS of radiation directivity azimuthal distribution $\sigma^{\prime}(x)$ (Fig. 2b).
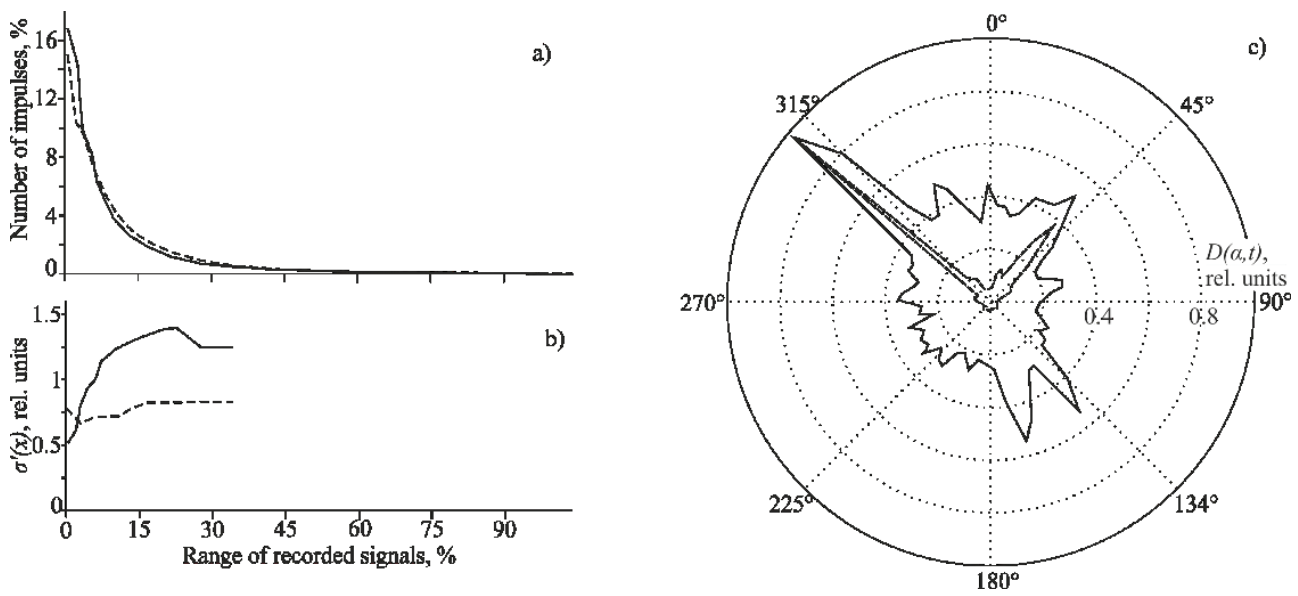

Fig. 2. Histogram of geoacoustic pulse amplitude distribution (a), curve $\sigma^{\prime}(x)$ the dependance of direcivity azimuthal distribution diagram RMS on signal amplitude (b), examples of diagrams of pulse repetition frequency azimuthal distribution (c). On the graphs (a) and (b), the solid lines indicate the diagrams for the periods of geoacoustic emission disturbances, dashed lines show that for the background periods. On the diagram (c), the solid line indicates the distribution diagram for the pulses with high amplitude, the dashed line is that with small amplitude. The curves (c) are in the relative scale for clarity.

Fig. 2 shows the results of estimation of the graphs $\sigma^{\prime}(x)$ for the geoacoustic pulses registered within 410 background periods twenty-four hour long and 77 periods with brightly distinguished disturbances of geoacoustic field before seismic events observed from 03.11.2008 to 30.01.2016. The calculation was made for the pulses with the magnitudes from 0 to $35 \%$ from the dynamic range of the registered signals. This interval was determined by the fact that the number of geoacoustic pulses exceeding this threshold is about $4-5 \%$ from the total number. Thus, their directivity azimuthal diagram is not accurate enough.

It is clear from the obtained results that the graph $\sigma^{\prime}(x)$ for the signals recorded before seismic events grows with the increase of geoacoustic pulse amplitude and becomes more than a unit at the point corresponding to $7 \%$ from the dynamic range of the registered signals. Thus, we can state that analysis of function $\sigma_{h}$ for the signals exceeding this value is the most informative and reasonable. It is interesting that the graph of $\sigma^{\prime}(x)$ corresponding to background periods does not have pronounced growth and does not exceed a unit at any point of the range. The obtained result was used to analyze geoacoustic emission properties before earthquakes.

It is logical to suppose that typical changes of geoacoustic emission are observed during the preparation of earthquakes the precursors of which manifest in the area where the signal is registered. The radius of this area can be estimated by the formula suggested by I.P. Dobrovol'skiy [5]: 


$$
\rho=10^{0.43 M} \mathrm{~km},
$$

where $M$ is the earthquake amplitude.

When the registration system is within the zone of precursor manifestation, the following inequality is fulfilled:

$$
\rho / r \geq 1
$$

where $r$ is the distance to an earthquake epicenter.

In the opposite case, when relation (3) is less than a unit, such earthquake precursors will not be recorded by the registering instrumentation.

This assumption was verified by a series of calculations by superposition method. For each group of calculation in a series, the earthquake occurrence times were chosen as the key dates, for which the relation $\rho / r$ was equal or exceeded the threshold $e_{i}$ where $e$ was measured from 0.3 to 1.9 with a step of 0.1 . On the whole, 17 separate calculation groups were derived in which from 2653 to 60 series of geoacoustic data were involved. The key dates were chosen in such a way as to exclude the superposition of close events. Moreover, we did not take into account the earthquakes which occurred at the distances of less than 35 $\mathrm{km}$ from the registration site and at the depths of more than $70 \mathrm{~km}$. Basically, it was done to ensure the estimation correctness of relation (3), which can result in high values as the distance from the sensor to an earthquake epicenter decreases.
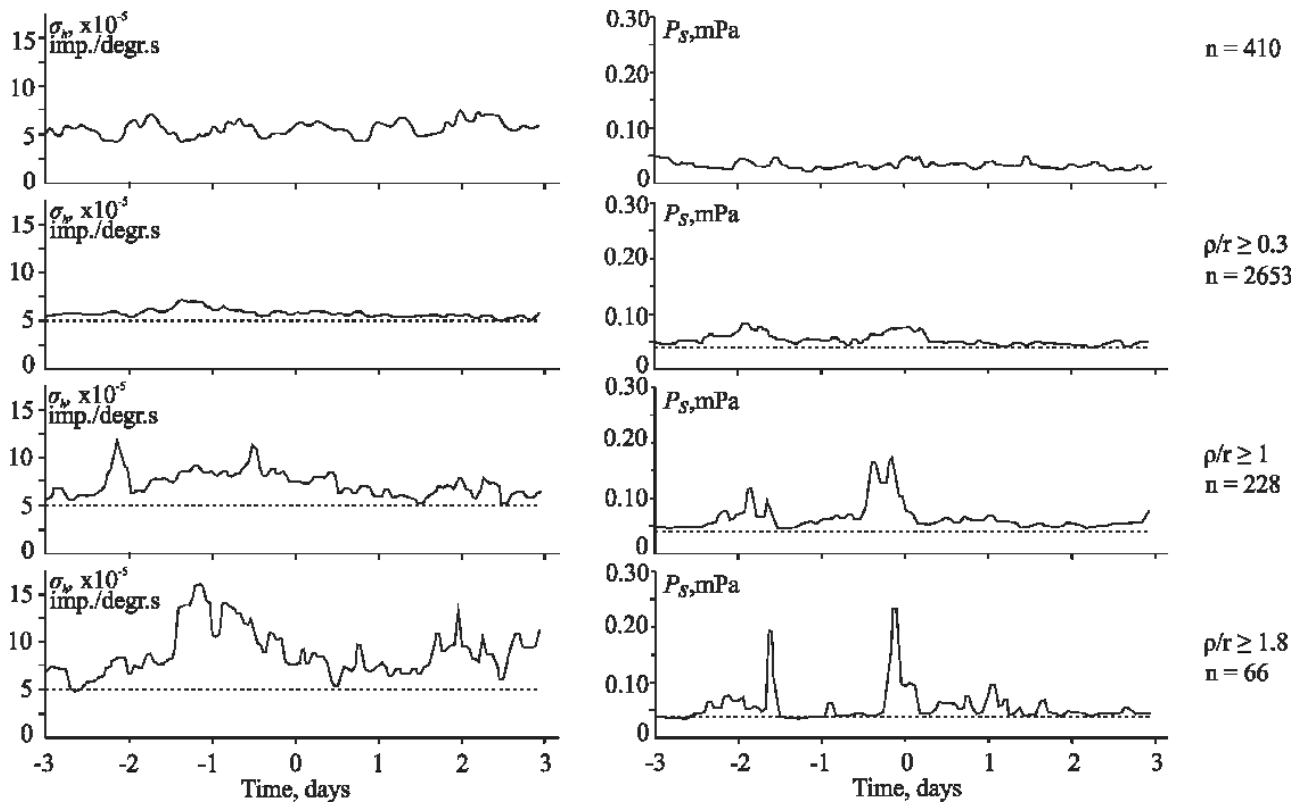

Fig. 3. Results of processing of geoacoustic signal by superposition method. The first graphs at the top correspond to background periods, the rest correspond to the periods of earthquake preparation. Dashed lines indicate the signal levels corresponding to background periods. The letter $\mathrm{n}$ indicates the data series number involved in the processing, $\sigma_{h}$ are azimuthal distribution diagram RMS, $P_{S}$ is total 1 -second acoustic pressure, $\rho$ is the precursor manifestation zone radius suggested by I.P.

Dobrovol'skiy, $r$ is the distance from the registration site to an earthquake epicenter.

For each data series, average graph of $\sigma_{h}(x)_{x=7 \%}$ was estimated as the most informative indicator of anomalous change of geoacoustic emission anisotropy (Fig. 3). Additionally, we determined the total (integral) value of acoustic pressure $P_{S}$ registered by the corresponding channel of a combined receiver in the frequency range of $2-6.5 \mathrm{kHz}$ [1]. The signal was 
stored in a window of $1 \mathrm{~s}$. For comparison, $\sigma_{h}(x)_{x=7 \%}$ and $P_{s}$ were calculated for 410 background periods twenty-four hour long.

It is clear from the diagrams that the averaged graph of $\sigma_{h}$ for the data series $\rho / r \geq 0.3$ almost does not differ from the curve plotted for the background period. The same refers to the graph of total acoustic pressure $P_{S}$. Thus, the earthquakes located at the distances, which exceed the precursor manifestation zone radius $\rho$ (2) by three times, do don affect the geoacoustic field in the region of measurements.

When analyzing the results of processing of the data series $\rho / r \geq 1$, we should note that the graph of $\sigma_{h}$ within the period from -2 to +1 day relatively the earthquake time significantly exceed the average value for the background. At the maximal point, the relation is almost double. A similar situation is observed on the graph of total acoustic pressure $P_{S}$, but here the background values are exceeded by 3.5 times. Thus, we can determine that significant change of geoacoustic emission properties is observed at the boundary of the precursor manifestation zone suggested by I.P. Dobrovol'skiy.

For systematization and further analysis of the calculation results, graphs of $\sigma_{s n r}$ and $P_{s n r}$ were made, similar to the relation signal/noise (Fig. 4)

$$
\sigma_{s n r}=\frac{\max \left(\sigma_{h S}(x)\right)}{\bar{\sigma}_{h n}(x)}, P_{s n r}=\frac{\max \left(P_{S S}\right)}{\bar{P}_{s n}},
$$

where $\max \left(\sigma_{h s}(x)\right)$ is the maximum value of $\sigma_{h}(x)_{x=7 \%}$ during disturbed periods, $\bar{\sigma}_{h n}(x)$ is the average value of $\sigma_{h}(x)_{x=7 \%}$ during background periods, $\max \left(P_{S S}\right)$ is $P_{S}$ maximum value during disturbances, $\bar{P}_{s n}$ is $P_{S}$ average value during background periods.

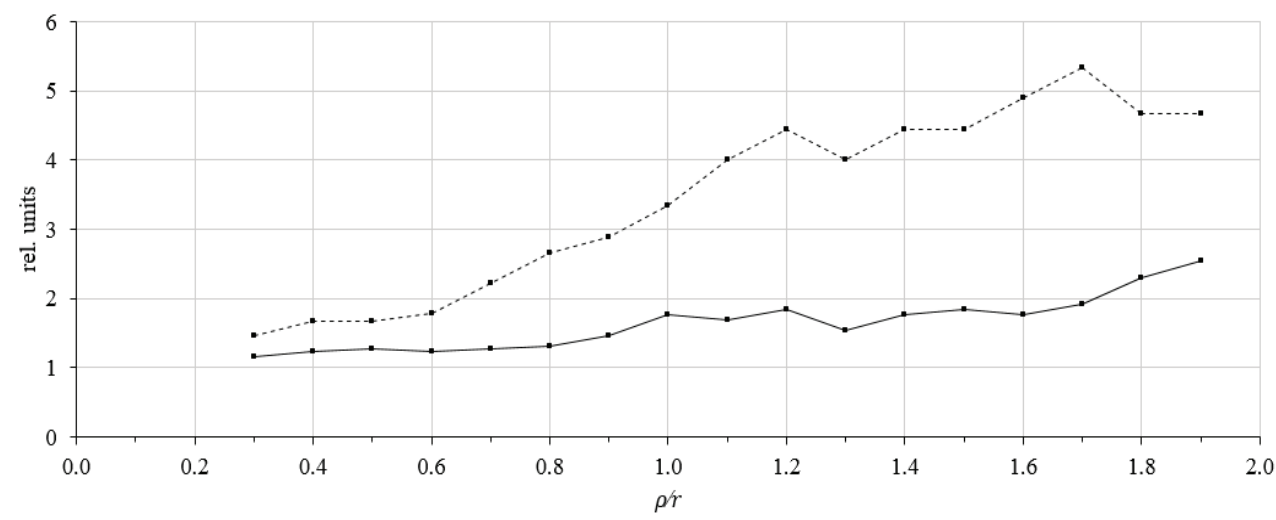

Fig. 4. Relative changes of diagram RMS of geoacoustic emission directivity azimuthal distribution (solid line) and total 1-second acoustic pressure (dashed line) depending on the relation of precursor manifestation zone radius suggested by I.P. Dobrovol'skiy to the distance to an earthquake epicenter $(\rho / r)$. The comparison was made with the background values.

Parts of the graphs close to 1 on the ordinate axis mean that geoacoustic emission anomalous change is not observed before an earthquake. Significant increase of $\sigma_{\text {snr }}$ graph begins from the value $\rho / r \geq 0.8$. Thus, geoacoustic emission anisotropy at the measurement point changes before earthquakes located $25 \%$ farther than we can determine by the formula suggested by I.P. Dobrovol'skiy. The total (integral) acoustic pressure $P_{s n r}$ grows from the point of 0.6 that corresponds to the precursor manifestation region increased by $65 \%$. 


\section{Conclusions}

The detailed analysis of geoacoustic data recorded in Kamchatka allowed us to determine that geoacoustic emission anisotropy grows as signal amplitude increases. This property was used to determine the effective parameters for signal filtration allowing us to increase the informative value of data statistic processing including the automated techniques. Based on the analysis of more than 2600 earthquakes, occurred from 01.01.2008 to 01.09.2016, by superposition method, the earthquake precursor manifestation zone radii were evaluated.

\section{References}

1. Yu.V. Marapulets, B.M. Shevtsov, I.A. Larionov, M.A. Mishchenko, A.O. Shcherbina, and A.A. Solodchuk, Russian Journal of Pacific Geology, 6, pp. 59-67 (2012).

2. V.A. Gordienko, T.V. Gordienko, N.V. Krasnopistsev, A.V. Kuptsov, I.A. Larionov, Yu.V. Marapulets, A.N. Rutenko, B.M. Shevtsov, Akusticheskij zhurnal, 1, pp. 97-109 (2008).

3. Yu.V. Marapulets, A.O. Shcherbina, Electronic Journal Technical Acoustics, 14 (2008).

4. M.A. Mishchenko, Bulletin of KRAESC. Phy. \& Math. Sci., 1, pp. 57-65 (2011).

5. I.P. Dobrovol'sky, S.I. Zubkov, V.I. Myachkin, Modeling of earthquake processes. Nauka Publishers, pp. 7-44 (1980). 